\title{
ANALYSIS ON PRODUCT TECHNICAL RISK WITH BAYESIAN BELIEF NETWORKS
}

\author{
Ming Chen ${ }^{1}$,Yun Chen ${ }^{2}$, Bingsen Chen ${ }^{1}$, Qun Wang ${ }^{1}$ \\ 'Sino-German College of Applied Sciences, Tongji University, China; Email: \\ cdhawcm@yahoo.com.cn. ${ }^{2}$ School of Public Economy Administration, Shanghai University of \\ finance \& economics, China.
}

Abstract: With the development of the science and technology, products are more and more complex, e.g. more functions integrated into one product, abilities of products are stronger and stronger, degrees of automatization and intelligentizing are higher and higher. In order to develop products successful, we must analyze product technical risk systematically and comprehensively. All products' behaviors that don't satisfy their functions are function failures, i.e. product technical risk. The sources of causing risk are the products' structures. We anticipate the behaviors of the target product, seek the potential function failures, and analyze the causes, and then set up BBN model. With this methodology, we have analyzed the product technical risk of a microelectronic equipment.

Key words: Product developing, Product technical risk, Bayesian belief networks.

\section{INTRODUCTION}

With the development of the science and technology, people have been improving their living and working situations constantly. The requirements of products that serve people are more and more, e.g. multiple functions are integrated into a product, abilities of system are more and more powerful, degrees of automatization and intelligentizing are very highly. It makes required products more and more complex.

Frequently, Design indexes of are diversity and some indexes are not consistent with the others. The target product consists of massive elements.

Please use the following format when citing this chapter: Chen, Ming, Chen, Yun, Chen, Bingsen, Wang, Qun, 2006, in International Federation for Information Processing (IFIP), Volume 207, Knowledge Enterprise: Intelligent Strategies In Product Design, Manufacturing, and Management, eds. K. Wang, Kovacs G., Wozny M., Fang M., (Boston: Springer), pp. 244-249. 
And the product's interfaces are multitudinous and complex. Most products are involved into many fields, e.g. mechanical engineering, electronic engineering, optical engineering etc. Designers lack of the experiences of usage of the advanced technology that are used in product developing.

In order to develop products successful, we must analyze product technical risk.

There are some ways to assess the risk, for example, fuzzy mathematics and neuron network, but they can't solve the essential problem of risk assessment: reasoning integrated with experts' knowledge based on uncertain information.

Bayesian Belief Network is a kind of expert system that can describe uncertain information. It can reflect continuity and accumulation of risk assessment. The consistency of time can't be realized with non-memorable methods, for example, methods based on rules and neuron network. Because of reliability in mathematics, Bayesian Belief Network can be changed into standard model which can express human thinking and reasoning process, while the traditional expert system is entirely oriented to tasks, and different tasks have their own models and they lake of catholicity.

The Product Technical Risk is modeled with Bayesian Belief Networks, constitutes assessing arithmetic, simulates and analyzes the technical risk.

\section{FUNCTION, STRUCTURE AND BEHAVIOR}

\subsection{Product system}

Products are entities that provide customers utilities and interests. Largescale products are systems composed of controls, units and interactions

Controls in product can be on product's level, on sub-systems' level or components' level. Units are non-control elements in product. Interactions are expressed that product's elements affect one other.

Any system has its own functions and shows its behaviors outside. System's behavior is process of realize relevant function, system's function is goal, results or constraints of behaviors. System's structure is total relations of elements in some system; it is a carrier of functions and behaviors.

\subsection{Method of analysis on product technical risk}

Owing to the complexity of systems in large-scale products, we must 
start analysis from the sources of product development: customers (Figure 1). All products' behaviors that don't satisfy their functions are function failure, i.e. product technical risk. The sources of causing risk are the products' structures. From the customers' requirements, the products' functions are acquired. According the functions, the structures are designed and after developing, the target product is manufactured. Analyzing the product's behaviors, we can judge whether or not the product satisfies the functions. We anticipate its behaviors, seek the potential function failure, i.e. product technical risk, and analyze the causes, i.e. its structure, and then set up Cause-and-Effect Chain, i.e. BBN model.

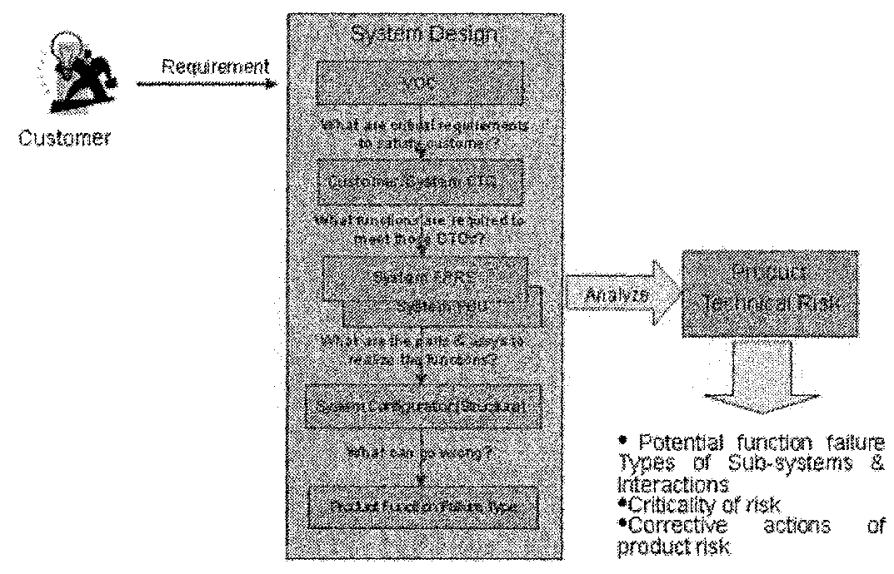

Figure 1. Method of product technical risk analysis

\section{MODEL OF PRODUCT TECHNICAL RISK}

\subsection{VOC, CTQ, FPRS and FBD}

The voice of the customer (VOC) is a process used to capture the requirements/feedback from the customer (internal or external) to provide the customers with the best in service/product quality. This process is all about being proactive and constantly innovative to capture the changing requirements of the customers with time.

Customer critical to quality (CTQ) is the key measurable characteristic of top level product whose performance standards or specification limits must 
be met in order to satisfy the customer. It aligns improvement or design efforts with customer requirements.

Starting with the customer's problem as outlined in the Customer/System CTQ, the Functional Performance Requirement Spec (FPRS) defines a product's functionality, usability, reliability, performance, supportability, availability, localizability, and other requirements. It describes the functions and performance levels that the proposed product should have to successfully address that problem.

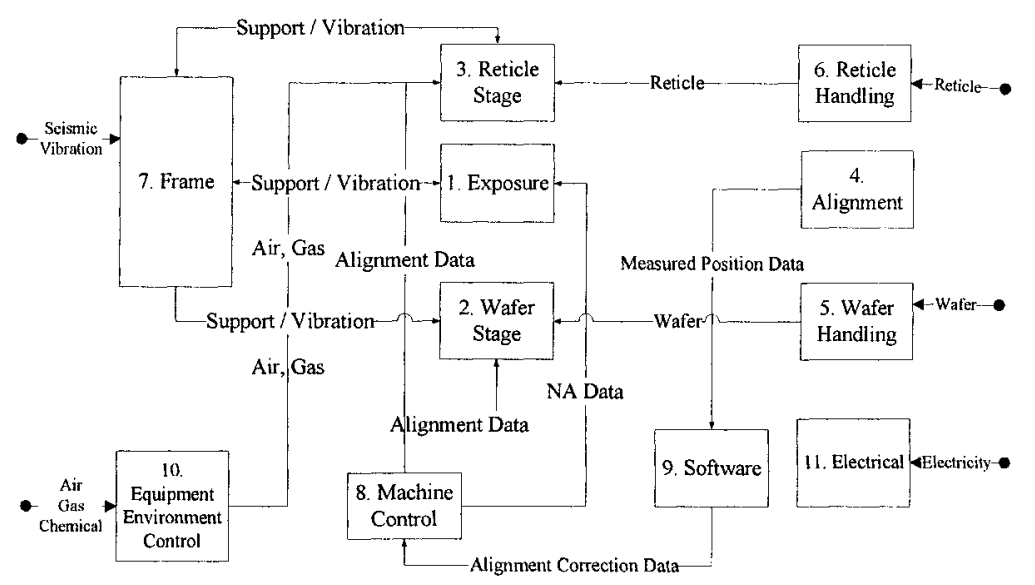

Figure 2. FBD of Step \& Scanner

Functional Block Diagram (FBD) is used to show how the different subsystems / parts interact with one another to verify the critical path. We prepare FBD in 3 different levels: system level FBD, sub-system level FBD and unit FBD. Figure 2 is a sample which shows the System Level of 300mm, 193 ArF, 130nm Step \& Scanner.

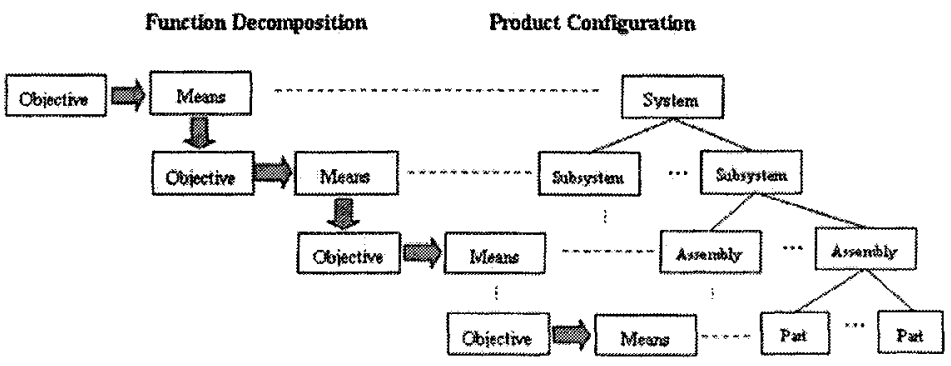

Figure 3. Function decomposition and product configuration 


\subsection{Product System Configuration}

The functions of the target product decompose some objectives and means on the system level. We decide which structures on this level according to the means. These means on this level are the objectives on the next level. We break them to some means. Then the structures of subsystem are designed. In this way, all the system is developed (Figure 3).

\subsection{BBN Model}

A BBN model is a directed acyclic graph (DAG), which is defined to consist of the qualitative and quantitative relationships. Qualitative Relationships include a set of random or deterministic variables and a set of directed edges or arcs. Quantitative Relationships include a set of root and conditional probabilities.

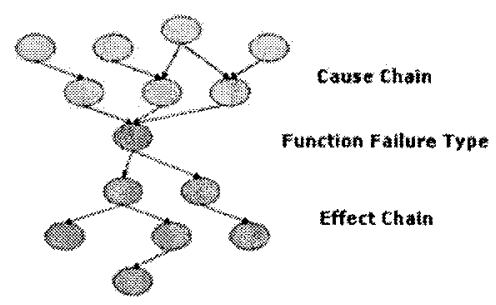

Figure 4. BBN model

Cause-and-Effect chain is a BBN model of the product risk analysis. Every function failure has its own model. It's easy to find causes that result in the function failure, and effect that the failure gives.

First we list the function failure, then list the phenomena occurs directly if the function failure appears, analyze the further effects and get effect chain. After that, we analyze the causes that result in the function failure, and analyze the further causes and get cause chain (Figure 4).

\section{CASE STUDY}

$300 \mathrm{~mm}, 193 \mathrm{ArF}, 130 \mathrm{~nm}$ Step \& Scanner is a kind of large-scale complex product which is related to mechanical engineering, electronic engineering, optical engineering, control engineering etc. We must analyze its technical risk during developing. With this methodology, we have analyzed the potential function failures. There are 20 function failure type, 
for example, $\mathrm{CD}$ resolution failure, $\mathrm{CD}$ uniformity failure etc. With the $\mathrm{BBN}$ model, we calculated the subsystem technical risk (Figure 5). We took some measures to improve the design and the risk has been reduced.

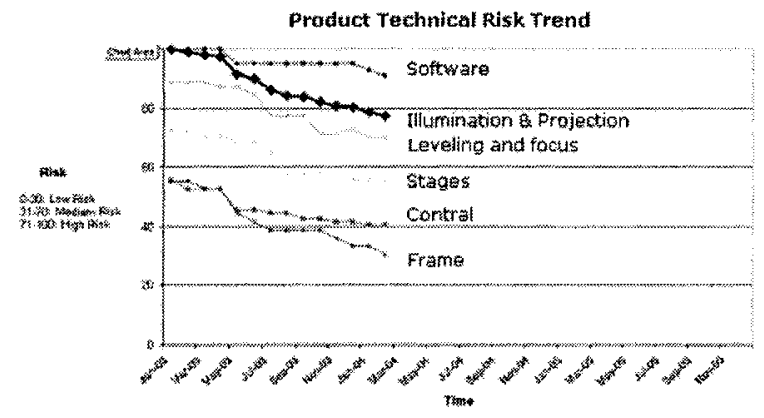

Figure 5. The product technical risk trend of the step \& scanner

\section{CONCLUSION}

In order to develop large-scale complex products successful, we must analyze product technical risk systematically. Product technical risk is its function failure. We analyze the behaviors of the target product and anticipate the potential function, i.e. failure product technical risk, and then analyze the causes, i.e. structure. In this way, its BBN model will be set up. We calculate the risk, and then it can guide us to take corrective actions in order.

\section{REFERENCES}

1. Chen Ming, Chen Yun, Chen Bingsen. Management of technical risk in the development of complex products. Manufacturing Automation 2005; 10:pp. 6-8.

2. Wang Guohua, Chen Ming, Chen Yun. Fuzzy Synthetized Estimating of Technical Risk of Complex Products. Mechatronic 2006; 1 :

3. Burton Hoyt Lee. Failure modes and effects analysis with Bayesian belief networks: bridging the design-diagnosis modeling gap. Dissertation of Stanford University 2002.

4. Elaine M.Hall. Managing Risk-Methods for Software Systems Development. Bosten: Addison-Wesley 1998

5. Wang Lianchen. Engineering System Theory. Beijing: China Astronautics publishing House, 2002

6. Eigenworx and ConneXwave. Advanced FMEA. SMEE 2003

7. ConneXwave. Managing Complex Projects Plan. SMEE 2003 